\title{
Light from Shattered Worlds: Debris from Giant Impacts
}

\author{
Alan P. Jackson ${ }^{1 \dagger}$, Mark C. Wyatt ${ }^{1}$, William R. F. Dent ${ }^{2}$ \\ and Aki Roberge ${ }^{3}$ \\ ${ }^{1}$ Institute of Astronomy, University of Cambridge, Madingley Road, Cambridge, CB3 0HA, UK \\ ${ }^{2}$ Joint ALMA Observatory, Alonso de Córdova 3107, Vitacura - Santiago, Chile \\ ${ }^{3}$ Goddard Space Flight Centre, 8800 Greenbelt Road, Greenbelt, Maryland, 20771, USA \\ ${ }^{\dagger}$ email: ajackson@ast.cam.ac.uk
}

\begin{abstract}
Large impacts in the outer parts of a planetary system will produce debris discs that display a strong, distinctive, asymmetry, which will last for $10^{5}$ year timescales. Debris resulting from a large impact may be able to explain the asymmetries in some known debris discs that have hitherto been difficult to understand.
\end{abstract}

Keywords. circumstellar matter, planetary systems, planetary systems: formation

\section{Introduction}

Impacts between planetary embryos drive the final stage of terrestrial planet formation (Kokubo \& Ida 1998). Impacts can be erosive or destructive (Agnor \& Asphaug 2004), and even efficiently accreting impacts produce debris equal to $\gtrsim 1$ per cent the mass of the colliding bodies. Giant impacts occur not only in the terrestrial zone but anywhere in a planetary system; Pluto-Charon (Canup 2011) and Haumea (Brown et al. 2007) in the Kuiper belt are both believed to have experienced large impacts. Debris production through giant impacts is thus an implicit component of planetary system formation.

Jackson \& Wyatt (2012) showed that debris produced in a Moon-forming like event around a nearby star should be detectable for tens of millions of years by typical Spitzer $24 \mu \mathrm{m}$ surveys. Detectable lifetime varies with the size of the largest fragments, but is over a million years even if these are only a few kilometres in size. The Moon-forming impact is a fairly low debris production event (Stewart \& Leinhardt 2012), and thus this is a reasonable baseline level of expected debris from planetary scale giant impacts. In the outer reaches of a planetary system orbital timescales are much slower, and collision velocities generally lower, and as such the lifetime of a debris disc of constant mass will increase as its semi-major axis is increased. Debris discs resulting from giant impacts in the outer reaches of a planetary system may thus be very long lived.

\section{Disc morphologies}

Since all debris fragments in a giant impact debris disc are produced at the original impact point, the orbits of these fragments must, at least initially, pass through the impact point. As a result the disc will have a strong asymmetry, which lasts for typically around 1000 orbits before precession of the debris orbits smears it out. In the terrestrial zone this leads to asymmetry lifetimes of $\sim 1000$ years, but in the outer regions the asymmetry can last for over $10^{5}$ years and, around a nearby star, can be readily resolved by modern instruments. 
In Fig. 1 we show an example of a debris disc produced by an impact at 85AU from the host star, both face-on and at several edge-on viewing angles, at $10^{5} \mathrm{yr}$ after the impact. Dynamical evolution is followed using the MERCurY N-body package (Chambers 1999). The width of the debris ring is controlled by the velocity dispersion of the debris, in Fig. 1 we use an isotropic dispersion of $4 \mathrm{~km} \mathrm{~s}^{-1}$; appropriate for roughly Mars size impactors. The progenitor is assumed to have been on a circular orbit. We also assume there are no massive ( $\sim$ Jupiter mass) planets nearby, but they may exist elsewhere in the system, e.g. the $7 M_{J} \beta$-Pic. b at $8.5 \mathrm{AU}$, without disrupting the disc. Bodies equivalent to the impactors can exist within the disc itself. These could be additional members of a primordial population, or the largest remnants of the impact.

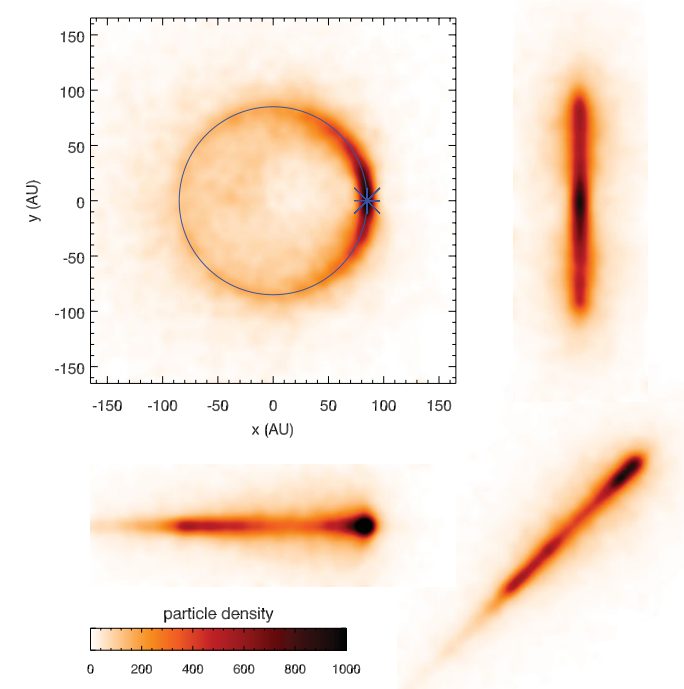

Figure 1. Images of a debris disk generated by a giant impact occuring at $85 \mathrm{AU}$ from the host star at $10^{5} \mathrm{yr}$ after impact. Resolution is $10 \mathrm{AU}(0.5 "$ at $20 \mathrm{pc})$. At top left we show a face-on view of the disk, with impact-point and progenitor body orbit indicated. Arranged around this we show edge-on views of the disk. Clockwise from top right the lines of sight are at $0^{\circ}$, $45^{\circ}$, and $90^{\circ}$ to the impact-point star line. Spatial and colour scales are consistent between images, with the exception that the density in the face-on image is enhanced by a factor of 2 to aid visibility.

For impacts in regions where ambient temperature is below $50 \mathrm{~K}$ and bodies contain significant quantities of CO ice, collisional grinding at the impact point may also produce a comet-like tail of $\mathrm{CO}$ gas that decays as it moves away from the impact point due to photo-dissociation, producing an even stronger asymmetry.

\section{Conclusions}

Dust from giant impacts is bright and detectable for ten million year timescales. Impacts in the outer reaches of a system will display distinctive asymmetries that last for $10^{5}$ year timescales and can be resolved around nearby stars. A number of known debris discs, such as the well known disc of Beta Pictoris, display large asymmetries that may be explainable as the result of a large impact, and with new facilities such as ALMA the number of such discs is only likely to increase.

\section{References}

Agnor C. \& Asphaug E., 2004, ApJL, 613, L157

Brown M. E., Barkume K. M., Ragozzine D., \& Schaller E. L., 2007, Nature, 446, 294

Canup R. M., 2011, AJ, 141, 35

Chambers J. E., 1999, MNRAS, 304, 793

Jackson A. P. \& Wyatt M. C., 2012, MNRAS, 425, 657

Kokubo E. \& Ida S., 1998, Icarus, 131, 171

Stewart S. T. \& Leinhardt Z. M., 2012, ApJ, 751, 32 